\title{
Genome-wide association and epistatic scan for unravelling the genetic architecture of complex traits and their practical applications in a breeding program
}

\author{
Sehgal D. ${ }^{1 *}$, Rosyara U. ${ }^{1}$, Mondal S. ${ }^{1}$, Singh R. ${ }^{1}$, Poland J. ${ }^{2}$, Dreisigacker S. ${ }^{1}$ \\ ${ }^{1}$ International Center for Maize and Wheat Improvement (CIMMYT), Carretera Méx-Veracruz, El Batán, \\ Texcoco, México \\ ${ }^{2}$ Kansas State University, Manhattan, Kansas, USA \\ *e-mail:d.sehgal@cgiar.org
}

Genome wide association mapping in conjunction with comprehensive epistatic scans of the genome can help unravelling the genetic architecture of complex traits such as grain yield and yield stability. Important genomic regions identified this way can propel common genomics-assisted breeding strategies implemented in a breeding program such as marker-assisted selection (MAS) and genomic selection (GS). We conducted in-depth investigation on the above on a large set of seven cohorts of advanced bread wheat lines $(n=6,518)$ within CIMMYT elite yield trials, which were genotyped with genotyping-by-sequencing markers and phenotyped under contrasting (irrigated and stress) environments. Haplotype-based genome wide association mapping identified a repertoire of beneficial alleles for GY and yield stability (superiority index $P i$ ) in CIMMYT germplasm on chromosomes 1A, 1B, 2B, 3B, 4A, 4B, 5B, 6B and 7B with allelic effects ranging from 2 to $10 \%$. Epistatic interactions contributed to an additional 5 to $9 \%$ variation on average. Stepwise regression of these genomic regions unraveled the best combination of alleles that can be used for MAS. We also explored whether integrating a subset of the consistent associations as fixed variables in prediction models improves prediction accuracy for GY and yield stability. For GY, the model led to up to $10 \%$ increase in prediction accuracy in cross-validation. Lines have been identified with different combinations of beneficial alleles to be included in crossing and line development schemes. 